\title{
Pressure-Interatomic Separation-Temperature Relationship of Alkali Halides
}

\author{
Q. LIU* \\ School of Physics and Material Science, Anhui University, Hefei 230039, China
}

(Received November 18, 2010; in final form February 8, 2011)

\begin{abstract}
A new method for the determination of pressure-interatomic separation-temperature relationship is investigated and applied for some alkali halides. The method is developed by using the Mie-Gruneisen equation of state and the Anderson thermal pressure and an ionic model based on Harrison's treatment of overlap repulsive potential which takes into account the interactions up to second neighbors. It is found that the new method yields satisfactory results in agreement with the available experimental data.
\end{abstract}

PACS: 64.30.-t, 65.40.De

\section{Introduction}

The understanding of the ionic solids at nonambient conditions is an integral part of physical sciences. Material scientists, solid state physicists, chemists, and solid earth geoscientists are routinely confronted with problems involving ionic solids at high pressure and high temperature [1]. In order to understand the behavior of ionic solids under the effect of high pressure and high temperature, considerable efforts have been made to determine the relationship between interatomic separation and temperature for alkali halides [2-7]. Moreover, at high pressure, relatively fewer efforts have been made.

The purpose of present paper is to develop a simple method for the determination of pressure-interatomic separation-temperature relationship. The calculations are performed within the framework of the MieGruneisen equation of state and Anderson thermal pressure [1]. The potential model considers: (i) the long range electrostatic interactions in terms of Madelung's energy; (ii) the short range overlap repulsive energy between nearest neighbors and next neighbors by adopting the analytical potential form derived by Harrison [8] based on quantum mechanical considerations; and (iii) the van der Waals dipole-dipole and dipole-quadrupole interactions. The method of analysis is described in Sect. 2. The results obtained are discussed and compared with the available experimental data for some alkali halides in Sect. 3.

\section{Theory and method of calculation}

The Mie-Gruneisen equation of state (EOS) can be expressed as follows [9]:

* e-mail: liuquananhui@yahoo.com.cn

$$
P=-\frac{\mathrm{d} \phi}{\mathrm{d} V}+P_{\mathrm{th}},
$$

where $\phi$ is the lattice potential energy and $V$ is the volume. In Eq. (1), the first term on right hand is the pressure due to volume dependence of electronic ground state energy (static pressure) and the second term is pressure due to vibration of nuclei (thermal pressure). $P$ is the externally applied pressure. The correct expression for thermal pressure $P_{\text {th }}$ can be taken from calculus as follows:

$$
\left(\frac{\partial P}{\partial T}\right)_{V}=\left(\frac{\partial P_{\mathrm{th}}}{\partial T}\right)_{V}=\alpha B_{T}
$$

where $T$ is the temperature, $\alpha$ is the coefficient of volume thermal expansion and $B_{T}$ is the isothermal bulk modulus. Upon integration we get

$$
P_{\mathrm{th}}=\int_{0}^{T}\left(\alpha B_{T}\right) \mathrm{d} T .
$$

This becomes $T \alpha B_{T}$ if the product $\alpha B_{T}$ is independent of $T$. This is approximately true above the Debye temperature $\theta_{\mathrm{D}}$ [10]. However, Anderson [1] pointed out that the thermal pressure should be dependent on volume compression. It means that the product $\alpha B_{T}$ may not be a constant, so Eq. (3) should be modified as [1]:

$$
P_{\mathrm{th}}=\left[\alpha_{0} B_{T_{0}}+\left(\frac{\partial B_{T}}{\partial T}\right)_{V} \ln \frac{V_{0}}{V}\right] T,
$$

where $\alpha_{0}$ and $B_{T_{0}}$ are the values of $\alpha$ and $B_{T}$ at reference temperature $T_{0}, V_{0}$ - the volume at zero pressure and reference temperature $T_{0}$. Substituting Eq. (4) into Eq. (1), we derived the following EOS:

$$
P=-\frac{\mathrm{d} \phi}{\mathrm{d} V}+\left[\alpha_{0} B_{T_{0}}+\left(\frac{\partial B_{T}}{\partial T}\right)_{V} \ln \frac{V_{0}}{V}\right] T .
$$

Now using the relation $V=x r^{3}$, Eq. (5) can be rewritten as follows: 


$$
P=-\frac{1}{3 x r^{2}} \frac{\mathrm{d} \phi}{\mathrm{d} r}+\left[\alpha_{0} B_{T_{0}}+\left(\frac{\partial B_{T}}{\partial T}\right)_{V} \ln \left(\frac{r_{0}}{r}\right)^{3}\right] T,
$$

where $x$ is a constant which depends on the structure of solid. $x$ equals 2 for NaCl-type and 1.54 for CsCl-type structures, and $r$ is the interatomic separation.

For the calculation of $\phi$, we use Harrison's potential form $[8]$ for the overlap repulsive energy and also consider van der Waals (vdW) dipole-dipole and dipolequadrupole interactions. Within the framework of Harrison's model the total lattice potential energy $\phi$ is expressed as follows:

$$
\phi=-\frac{a_{\mathrm{M}} Z^{2} e^{2}}{r}-\frac{C}{r^{6}}-\frac{D}{r^{8}}+\phi_{\text {rep }},
$$

where the first term on the right-hand side of Eq. (7) is the electrostatic Coulomb energy with $a_{\mathrm{M}}$ as Madelung's constant which is equal to 1.7476 for NaCl-type structures and 1.7627 for $\mathrm{CsCl}$-type structures, $e$ - the charge of the electron, and $Z$ - the valency. The second and third terms are van der Waals (vdW) dipole-dipole and dipole-quadrupole energies. The constants $C$ and $D$ are related to the dipole-dipole $\left(c_{i j}\right)$ and dipole-quadrupole $\left(d_{i j}\right)$ interaction coefficients as follows [11]:

$$
\begin{aligned}
& C=S_{+-} c_{+-}+S_{++} c_{++}+S_{-} c_{-}, \\
& D=T_{+-} d_{+-}+T_{++} d_{++}+T_{-} d_{-},
\end{aligned}
$$

where $S_{i j}$ and $T_{i j}$ are lattice sums which had been given by Tosi [12]. The subscripts,,+-++-- represent the cation-anion, cation-cation, anion-anion interactions, respectively. The values of $c_{i j}$ and $d_{i j}$ are obtained from the Kirkwood-Muller formulae given below [11]:

$$
\begin{aligned}
c_{i j} & =-\frac{6 m c^{2}}{N} X_{i} X_{j}\left(\frac{X_{i}}{a_{i}}+\frac{x_{j}}{a_{j}}\right)^{-1}, \\
d_{i j} & =-\frac{9 m c^{2}}{N e^{2}} c_{i j}\left(\frac{X_{i}}{n_{i}}+\frac{X_{i}}{n_{j}}\right),
\end{aligned}
$$

where $c$ is the velocity of light, $a$ and $X$ are the polarisabilities and molar susceptibilities, respectively. $N$ is the Avogadro number. $n_{i}$ and $n_{j}$ are the number of outmost electrons. The values of $C$ and $D$ calculated from Eqs. (8)-(11) were used in the present work.

For the overlap repulsive energy, we use the analytical form proposed by Harrison [8]. The normalized charge density as used by Harrison from quantum mechanical consideration is

$$
n(r)=\frac{6 \mu^{3}}{\pi} \exp (-2 \mu r),
$$

where $\mu$ is related to the valence $p$ and state energy $\left(\varepsilon_{p}\right)$ as follows:

$$
\varepsilon_{p}=-\frac{\hbar^{2} \mu^{2}}{2 m},
$$

where $\hbar$ is Planck's constant $h$ divided by $2 \pi$ and $m$ is the mass of an electron. The total overlap interaction has been considered as arising from three contributions, viz., (i) the kinetic energy, (ii) the exchange energy and (iii) the Coulombian energy of electrons in the overlap region. These contributions to the overlap repulsive potential are given below [8]:

$$
\begin{aligned}
& \phi^{\mathrm{ke}}=70.8 \varepsilon_{p} \mu r \exp \left(-\frac{5 \mu r}{3}\right), \\
& \phi^{\mathrm{ex}}=-2.728 e^{2} \mu^{2} r \exp \left(-\frac{4 \mu r}{3}\right), \\
& \phi^{\mathrm{coul}}=-6 e^{2} \mu^{3} r^{2} \exp (-2 \mu r) .
\end{aligned}
$$

Harrison [8] assumed that the total overlap interaction is of the form of the kinetic energy term and can be expressed by an equation similar to that given by Eq. (14). So we can write the overlap repulsive potential as follows:

$$
\begin{aligned}
& \phi_{\text {rep }}=\frac{n_{0} \hbar^{2}}{2 m}\left[M \bar{\mu}^{3} r \exp (-k \bar{\mu} r)\right. \\
& \left.\quad+\frac{1}{2} M^{\prime} \mu_{1}^{3} r^{\prime} \exp \left(-k \mu_{1} r^{\prime}\right)+\frac{1}{2} M^{\prime} \mu_{2}^{3} r^{\prime} \exp \left(-k \mu_{2} r^{\prime}\right)\right] .
\end{aligned}
$$

For NaCl-type structures, $M=6$ and $M^{\prime}=12$ are the numbers of first and second neighbor ions. Similarly, $r$ and $r^{\prime}=\sqrt{2} r$ are the first and second nearest neighbor distances. For CsCl-type structures $M=8, M^{\prime}=6$, and $r^{\prime}=(2 / \sqrt{3}) r$. The values of $\mu_{1}$ and $\mu_{2}$ are calculated using the valence $p$ state energy $\left(\varepsilon_{p}\right)$ given by Eq. (13), and $\bar{\mu}$ is the arithmetic average of $\mu_{1}$ and $\mu_{2}$ for the cation and anion.

In order to calculate lattice potential energy $\phi$ from Eq. (7), there remain only two unknown parameters, viz. $n_{0}$ and $k$ which are obtained from the equilibrium condition and the following relations:

$$
\begin{aligned}
& \left(\frac{\mathrm{d} \phi}{\mathrm{d} r}\right)_{r=r_{0}}=0, \\
& \left(\frac{\mathrm{d}^{2} \phi}{\mathrm{d} r^{2}}\right)_{r=r_{0}}=9 x r_{0} B_{T_{0}} .
\end{aligned}
$$

The potential parameters thus calculated are assumed to be independent of pressure. The input data required are given in Table I. Now, the values of $\mathrm{d} \phi / \mathrm{d} r$, the first derivative of the lattice energy can be calculated using Eq. (7) at any value of $r$, and finally the pressure $P$ is calculated using Eq. (6) at different temperature. The values of pressure thus calculated for different values of $r$ are given in Tables II-VI along with the available experimental data. At $P=0$, Eq. (6) takes the following form:

$$
T=\frac{1}{3 x r^{2}} \frac{\mathrm{d} \phi}{\mathrm{d} r} /\left[\alpha_{0} B_{T_{0}}+\left(\frac{\partial B_{T}}{\partial T}\right)_{V} \ln \left(\frac{r_{0}}{r}\right)^{3}\right] .
$$

Now the values of $\mathrm{d} \phi / \mathrm{d} r$ are calculated for increasing $r$ and the corresponding temperature $T$ is evaluated from Eq. (20) for some alkali halides. The results obtained are reported in Table VII along with the available experimental data. 
Values of input parameters used in the present work [1, 11, and 13].

TABLE I

\begin{tabular}{c|c|c|c|c|c|c|c|c|c|c}
\hline \hline Crystal & $\begin{array}{c}r_{0} \\
{[\AA]}\end{array}$ & $\begin{array}{c}\alpha_{0} \\
{\left[10^{-4} \mathrm{~K}^{-1}\right]}\end{array}$ & $\begin{array}{c}B_{T_{0}} \\
{[\mathrm{kbar}]}\end{array}$ & $\left(\partial B_{T} / \partial T\right)_{V}$ & $\begin{array}{c}C \\
{\left[10^{-60} \mathrm{erg} \mathrm{\textrm {cm } ^ { 6 } ]}\right.}\end{array}$ & $\begin{array}{c}D \\
{\left[10^{-76} \mathrm{erg} \mathrm{cm}^{8}\right]}\end{array}$ & $n_{0}$ & $k$ & $\begin{array}{c}\mu_{1} \\
{\left[10 \mathrm{~nm}^{-1}\right]}\end{array}$ & $\begin{array}{c}\mu_{2} \\
{\left[10 \mathrm{~nm}^{-1}\right]}\end{array}$ \\
\hline $\mathrm{LiF}$ & 2.013 & 0.999 & 665 & -0.051 & 46 & 26 & 3 & 1.34 & 3.962 & 2.112 \\
$\mathrm{LiCl}$ & 2.570 & 1.32 & 297 & -0.027 & 317 & 221 & 11.6 & 1.41 & 3.962 & 1.799 \\
$\mathrm{LiBr}$ & 2.751 & 1.50 & 235 & -0.025 & 594 & 286 & 9.5 & 1.34 & 3.962 & 1.716 \\
$\mathrm{LiI}$ & 3.006 & 1.80 & 172 & -0.023 & 1265 & 584 & 61.6 & 1.57 & 3.962 & 1.619 \\
$\mathrm{NaF}$ & 2.317 & 0.96 & 465 & -0.025 & 139 & 98 & 11.5 & 1.54 & 3.087 & 2.112 \\
$\mathrm{NaCl}$ & 2.820 & 1.19 & 240 & -0.017 & 561 & 455 & 19.6 & 1.49 & 3.087 & 1.799 \\
$\mathrm{NaBr}$ & 2.989 & 1.26 & 199 & -0.016 & 930 & 537 & 30.7 & 1.52 & 3.087 & 1.716 \\
$\mathrm{NaI}$ & 3.236 & 1.37 & 151 & -0.013 & 1755 & 942 & 48.3 & 1.53 & 3.087 & 1.619 \\
$\mathrm{KCl}$ & 3.146 & 1.11 & 175 & -0.012 & 607 & 285 & 70.9 & 1.67 & 2.487 & 1.799 \\
$\mathrm{KBr}$ & 3.289 & 1.16 & 148 & -0.010 & 1854 & 962 & 112 & 1.71 & 2.487 & 1.716 \\
$\mathrm{KI}$ & 3.525 & 1.23 & 117 & -0.008 & 2745 & 1430 & 64.5 & 1.57 & 2.487 & 1.619 \\
$\mathrm{RbCl}$ & 2.570 & 1.32 & 297 & -0.011 & 317 & 221 & 11.6 & 1.41 & 3.962 & 1.799 \\
$\mathrm{RbBr}$ & 2.751 & 1.50 & 235 & -0.009 & 594 & 286 & 9.5 & 1.34 & 3.962 & 1.716 \\
$\mathrm{RbI}$ & 3.006 & 1.80 & 172 & -0.008 & 1265 & 584 & 61.6 & 1.57 & 3.962 & 1.619 \\
$\mathrm{CsCl}$ & 3.571 & 1.37 & 180 & -0.015 & 333 & 357 & 974 & 2.08 & 2.116 & 1.799
\end{tabular}

TABLE II

Values of pressure ( $P$ in kbar) vs. interatomic separation for LiF crystal at different temperatures. The available experimental data [15] are given within parentheses.

\begin{tabular}{c|c|c|c|c|c|c|c}
\hline \hline \multicolumn{2}{c|}{$298 \mathrm{~K}$} & \multicolumn{2}{c|}{$573 \mathrm{~K}$} & \multicolumn{2}{c|}{$873 \mathrm{~K}$} & \multicolumn{2}{c}{$1073 \mathrm{~K}$} \\
\hline$r$ & $P$ & $r$ & $P$ & $r$ & $P$ & $r$ & $P$ \\
\hline 2.013 & $0(0)$ & 2.035 & $0(0)$ & 2.066 & $0(0)$ & 2.093 & $0(0)$ \\
2.003 & $9.98(10)$ & 2.024 & $9.98(10)$ & 2.053 & $9.99(10)$ & 2.077 & $10.01(10)$ \\
1.994 & $19.94(20)$ & 2.014 & $19.87(20)$ & 2.041 & $19.91(20)$ & 2.063 & $19.94(20)$ \\
1.985 & $29.72(30)$ & 2.004 & $29.87(30)$ & 2.030 & $29.96(30)$ & 2.050 & $30.04(30)$ \\
1.977 & $39.84(40)$ & 1.995 & $39.64(40)$ & 2.019 & $39.84(40)$ & 2.037 & $39.91(40)$ \\
1.969 & $49.88(50)$ & 1.986 & $49.56(50)$ & 2.009 & $49.72(50)$ & 2.026 & $49.75(50)$ \\
1.961 & $59.59(60)$ & 1.977 & $59.77(60)$ & 1.999 & $59.47(60)$ & 2.015 & $59.79(60)$ \\
1.954 & $69.75(70)$ & 1.969 & $69.15(70)$ & 1.944 & $69.23(70)$ & 2.004 & $69.58(70)$ \\
1.946 & $79.46(80)$ & 1.961 & $78.99(80)$ & 1.982 & $79.45(80)$ & 1.994 & $79.46(80)$ \\
1.940 & $89.16(90)$ & 1.953 & $88.75(90)$ & 1.973 & $88.97(90)$ & 1.985 & $89.03(90)$
\end{tabular}

\section{Results and discussions}

In order to judge the influence of the volume independence of thermal pressure of presented results, we have compared the values of pressure obtained by Eq. (3) and Eq. (4) with the experimental data [14] for $\mathrm{NaCl}$ crystal at $573 \mathrm{~K}$ (Table VIII). We note that the values of $P$ calculated through Eq. (4) are in close agreement with experimental data. Thus, Eq. (4) proposed by the Anderson, seems to be more suitable for the thermal pressure than Eq. (3).
We have proposed a simple method to investigate the pressure-interatomic separation-temperature relationship in the wide range of temperatures and pressures. It is clear from Tables II-VII that the calculated values for all alkali halides are in good agreement with the experimental values [14-18], supporting the validity of the simple method.

For estimating the values of $\mathrm{d} \phi / \mathrm{d} r$ at $r$, we have used the potential energy expression given in the form of Eq. (7). Within the framework of this model, we have considered vdW dipole-dipole and dipole-quadrupole interactions. The dipole-dipole energy term, which arises 
TABLE III

Values of pressure $(P$ in kbar) vs. interatomic separation for $\mathrm{NaF}$ crystal at different temperatures. The available experimental data [15] are given within parentheses.

\begin{tabular}{c|c|c|c|c|c|c|c|c|c}
\hline \hline \multicolumn{2}{c|}{$298 \mathrm{~K}$} & \multicolumn{2}{c|}{$473 \mathrm{~K}$} & \multicolumn{2}{c|}{$673 \mathrm{~K}$} & \multicolumn{2}{|c|}{$873 \mathrm{~K}$} & \multicolumn{2}{c}{$1073 \mathrm{~K}$} \\
\hline$r$ & $P$ & $r$ & $P$ & $r$ & $P$ & $r$ & $P$ & $r$ & $P$ \\
\hline 2.317 & $0(0)$ & 2.331 & $0(0)$ & 2.349 & $0(0)$ & 2.369 & $0(0)$ & 2.392 & $0(0)$ \\
2.301 & $9.99(10)$ & 2.314 & $10.03(10)$ & 2.331 & $9.98(10)$ & 2.348 & $9.99(10)$ & 2.370 & $10.00(10)$ \\
2.286 & $19.91(20)$ & 2.299 & $19.89(20)$ & 2.314 & $19.94(20)$ & 2.330 & $19.95(20)$ & 2.349 & $19.98(20)$ \\
2.273 & $29.88(30)$ & 2.285 & $29.91(30)$ & 2.299 & $29.95(30)$ & 2.314 & $29.94(30)$ & 2.331 & $29.94(30)$ \\
2.260 & $39.82(40)$ & 2.272 & $39.71(40)$ & 2.285 & $39.88(40)$ & 2.299 & $39.92(40)$ & 2.315 & $39.96(40)$ \\
2.249 & $49.65(50)$ & 2.259 & $49.64(50)$ & 2.272 & $49.81(50)$ & 2.285 & $49.85(50)$ & 2.300 & $49.87(50)$ \\
2.238 & $59.47(60)$ & 2.248 & $59.51(60)$ & 2.260 & $59.60(60)$ & 2.273 & $59.71(60)$ & 2.286 & $59.84(60)$ \\
2.228 & $69.21(70)$ & 2.237 & $69.21(70)$ & 2.249 & $69.37(70)$ & 2.261 & $69.58(70)$ & 2.273 & $69.55(70)$ \\
2.218 & $78.74(80)$ & 2.226 & $78.96(80)$ & 2.238 & $79.11(80)$ & 2.250 & $79.30(80)$ & 2.261 & $79.37(80)$ \\
2.210 & $88.80(90)$ & 2.217 & $88.72(90)$ & 2.228 & $88.99(90)$ & 2.240 & $89.18(90)$ & 2.249 & $89.44(90)$
\end{tabular}

TABLE IV

Values of pressure ( $P$ in kbar) vs. interatomic separation for $\mathrm{NaCl}$ crystal at different temperatures. The available experimental data [14] are given within parentheses.

\begin{tabular}{c|c|c|c|c|c|c|c}
\hline \hline \multicolumn{2}{c|}{$298 \mathrm{~K}$} & \multicolumn{2}{c|}{$473 \mathrm{~K}$} & \multicolumn{2}{c|}{$673 \mathrm{~K}$} & \multicolumn{2}{c}{$773 \mathrm{~K}$} \\
\hline$r$ & $P$ & $r$ & $P$ & $r$ & $P$ & $r$ & $P$ \\
\hline 2.820 & $0(0)$ & 2.840 & $0(0)$ & 2.868 & $0(0)$ & 2.885 & $0(0)$ \\
2.801 & $5.0(5)$ & 2.819 & $5.0(5)$ & 2.844 & $5.0(5)$ & 2.839 & $5.0(5)$ \\
2.784 & $10.0(10)$ & 2.801 & $10.0(10)$ & 2.821 & $10.0(10)$ & 2.814 & $10.0(10)$ \\
2.769 & $14.9(15)$ & 2.785 & $14.9(15)$ & 2.804 & $14.9(15)$ & 2.796 & $14.8(15)$ \\
2.754 & $19.8(20)$ & 2.769 & $19.7(20)$ & 2.788 & $19.8(20)$ & 2.781 & $19.8(20)$ \\
2.740 & $24.6(25)$ & 2.755 & $24.6(25)$ & 2.771 & $24.5(25)$ & 2.763 & $24.6(25)$ \\
2.728 & $29.8(30)$ & 2.742 & $29.7(30)$ & 2.755 & $29.7(30)$ & 2.750 & $29.8(30)$ \\
2.717 & $34.6(35)$ & 2.730 & $34.5(35)$ & 2.745 & $34.7(35)$ & &
\end{tabular}

TABLE V

Values of pressure $(P$ in kbar) vs. interatomic separation for $\mathrm{CsCl}$ crystal at different temperatures. The available experimental data [15] are given within parentheses.

\begin{tabular}{c|c|c|c|c|c|c|c}
\hline \hline \multicolumn{2}{c|}{$298 \mathrm{~K}$} & \multicolumn{2}{c|}{$473 \mathrm{~K}$} & \multicolumn{2}{|c|}{$673 \mathrm{~K}$} & \multicolumn{2}{c}{$873 \mathrm{~K}$} \\
\hline$r$ & $P$ & $r$ & $P$ & $r$ & $P$ & $r$ & $P$ \\
\hline 3.571 & $0(0)$ & 3.603 & $0(0)$ & 3.646 & $0(0)$ & 3.696 & $0(0)$ \\
3.514 & $9.96(10)$ & 3.537 & $9.97(10)$ & 3.568 & $9.99(10)$ & 3.600 & $10.01(10)$ \\
3.468 & $19.91(20)$ & 3.485 & $19.92(20)$ & 3.509 & $19.25(20)$ & 3.532 & $19.37(20)$ \\
3.430 & $29.33(30)$ & 3.443 & $29.45(30)$ & 3.462 & $29.47(30)$ & 3.479 & $29.37(30)$ \\
3.397 & $38.98(40)$ & 3.407 & $39.41(40)$ & 3.422 & $39.29(40)$ & 3.435 & $39.50(40)$ \\
3.368 & $48.72(50)$ & 3.376 & $48.99(50)$ & 3.388 & $49.25(50)$ & 3.398 & $49.24(50)$ \\
3.343 & $58.97(60)$ & 3.348 & $59.02(60)$ & 3.358 & $58.97(60)$ & 3.366 & $59.13(60)$ \\
3.319 & $68.58(70)$ & 3.323 & $68.96(70)$ & 3.332 & $69.01(70)$ & 3.337 & $68.92(70)$ \\
3.298 & $78.66(80)$ & 3.301 & $78.49(80)$ & 3.307 & $78.58(80)$ & 3.311 & $79.56(80)$ \\
3.279 & $88.29(90)$ & 3.280 & $88.84(90)$ & 3.284 & $88.97(90)$ & 3.287 & $89.10(90)$
\end{tabular}


TABLE VI

Values of pressure ( $P$ in kbar) vs. interatomic separation for some alkali halides at room temperature. The available experimental data $[14,16]$ are given within parentheses.

\begin{tabular}{|c|c|c|c|c|c|}
\hline \multicolumn{2}{|c|}{$\mathrm{LiCl}$} & \multicolumn{2}{|c|}{$\mathrm{LiBr}$} & \multicolumn{2}{|c|}{ LiI } \\
\hline$r$ & $P$ & $r$ & $P$ & $r$ & $P$ \\
\hline 2.570 & $0(0)$ & 2.751 & $0(0)$ & 3.006 & $0(0)$ \\
\hline 2.557 & $5.0(5)$ & 2.733 & $5.0(5)$ & 2.978 & $5.0(5)$ \\
\hline 2.545 & 10.1(10) & 2.717 & $10.2(10)$ & 2.954 & $10.0(10)$ \\
\hline 2.533 & $15.2(15)$ & 2.700 & $15.2(15)$ & 2.931 & $15.1(15)$ \\
\hline 2.522 & $20.5(20)$ & 2.685 & $20.0(20)$ & 2.911 & $20.0(20)$ \\
\hline 2.511 & $25.5(25)$ & 2.672 & $24.9(25)$ & 2.893 & $25.1(25)$ \\
\hline 2.501 & $30.5(30)$ & 2.658 & $29.9(30)$ & 2.876 & $30.6(30)$ \\
\hline 2.491 & $35.6(35)$ & 2.646 & $34.7(35)$ & 2.860 & $35.5(35)$ \\
\hline 2.481 & $41.0(40)$ & 2.634 & $39.5(40)$ & 2.847 & $40.5(40)$ \\
\hline 2.473 & $45.8(45)$ & 2.624 & $44.3(45)$ & 2.833 & $45.5(45)$ \\
\hline \multicolumn{2}{|c|}{$\mathrm{NaBr}$} & \multicolumn{2}{|c|}{$\mathrm{NaI}$} & & \\
\hline$r$ & $P$ & $r$ & $P$ & & \\
\hline 2.989 & $0(0)$ & 3.237 & $0(0)$ & & \\
\hline 2.966 & $5.0(5)$ & 3.203 & $5.0(5)$ & & \\
\hline 2.945 & $10.0(10)$ & 3.173 & $9.9(10)$ & & \\
\hline 2.925 & $14.8(15)$ & 3.147 & $14.9(15)$ & & \\
\hline 2.908 & $19.9(20)$ & 3.124 & $19.9(20)$ & & \\
\hline 2.892 & $24.6(25)$ & 3.103 & $24.8(25)$ & & \\
\hline 2.877 & $29.7(30)$ & 3.084 & $29.8(30)$ & & \\
\hline 2.863 & $34.6(35)$ & 3.067 & $34.8(35)$ & & \\
\hline 2.849 & $39.9(40)$ & 3.049 & $39.9(40)$ & & \\
\hline 2.837 & $44.6(45)$ & 3.03 & $45.2(45)$ & & \\
\hline
\end{tabular}

from the interaction between induced dipole moments of different atoms, is actually the first term in an infinite series of rapidly converging terms. The dipole-quadrupole term is interpreted as arising from the interaction of a dipole moment on one atom with a quadrupole on the other. Actually, there exists a third term, which varies inversely as the tenth power of interatomic distance, which is called the quadrupole-quadrupole term. In the present article we discuss only the dipole-dipole and dipole-quadrupole interactions because the quadrupolequadrupole term is negligible in ionic crystals.

It should be mentioned that the overlap potential forms as given by Eq. (17) are based on Harrison's quantum mechanical formulation and differ from the traditional Born-Mayer exponential forms [19, 20] in some important aspects. First, the pre-exponential factors appearing in Eq. (17) also depend directly on the interionic separation whereas in the Born-Mayer exponential forms, only the exponential factors depend on $r$. Secondly, ionic radii have been introduced arbitrarily as adjustable parameters in the Born-Mayer exponential forms. On the other hand, in Eq. (17) the pre-exponential as well as exponential factors depend on fundamental factors like Planck's
TABLE VII

Values of temperature ( $T$ in $\mathrm{K})$ vs. interatomic separation for some alkali halides at atmospheric pressure $(P=0)$. The available experimental data $[17,18]$ are given within parentheses.

\begin{tabular}{c|c|c|c|c|c}
\hline \hline \multicolumn{2}{|c|}{$\mathrm{KCl}$} & \multicolumn{2}{|c|}{$\mathrm{KBr}$} & \multicolumn{2}{|c}{$\mathrm{KI}$} \\
\hline$r$ & $T$ & $r$ & $T$ & $r$ & $T$ \\
\hline 3.116 & $0(0)$ & 3.26 & $0(0)$ & 3.489 & $0(0)$ \\
3.12 & $39(39)$ & 3.28 & $173(175)$ & 3.50 & $88(90)$ \\
3.14 & $220(222)$ & 3.30 & $346(349)$ & 3.52 & $225(230)$ \\
3.16 & $391(395)$ & 3.32 & $507(512)$ & 3.54 & $349(355)$ \\
3.18 & $490(492)$ & 3.34 & $647(650)$ & 3.56 & $466(475)$ \\
3.20 & $641(645)$ & 3.36 & $777(780)$ & 3.58 & $569(576)$ \\
3.22 & $775(778)$ & 3.38 & $894(894)$ & 3.60 & $669(680)$ \\
3.24 & $893(894)$ & 3.40 & $1013(1015)$ & 3.62 & $754(760)$ \\
3.26 & $1000(1002)$ & & & 3.64 & $837(850)$ \\
3.27 & $1049(1052)$ & & & 3.66 & $908(915)$ \\
\hline & $\mathrm{RbCl}$ & \multicolumn{2}{|c|}{$\mathrm{RbBr}$} & \multicolumn{2}{c}{$\mathrm{RbI}$} \\
\hline$r$ & $T$ & $r$ & $T$ & $r$ & $T$ \\
\hline 3.259 & $0(0)$ & 3.41 & $0(0)$ & 3.628 & $0(0)$ \\
3.26 & $36(40)$ & 3.42 & $92(95)$ & 3.64 & $101(105)$ \\
3.28 & $211(215)$ & 3.44 & $231(235)$ & 3.66 & $206(205)$ \\
3.30 & $316(320)$ & 3.46 & $324(330)$ & 3.68 & $326(315)$ \\
3.32 & $421(430)$ & 3.48 & $418(425)$ & 3.70 & $402(410)$ \\
3.34 & $539(550)$ & 3.50 & $508(512)$ & 3.72 & $470(475)$ \\
3.36 & $629(635)$ & 3.52 & $693(699)$ & 3.74 & $542(545)$ \\
3.38 & $754(770)$ & 3.54 & $784(788)$ & 3.76 & $613(615)$ \\
3.40 & $879(900)$ & 3.56 & $872(880)$ & 3.78 & $781(780)$ \\
3.42 & $1008(1020)$ & 3.58 & $961(970)$ & 3.80 & $850(845)$
\end{tabular}

TABLE VIII

Values of pressure ( $P$ in kbar) obtained by Eq. (3) (column (a)) and Eq. (4) (column (b)) for NaCl crystal at $573 \mathrm{~K}$. The experimental values (column (c)) are taken from Ref. [14].

\begin{tabular}{c|c|c|c}
\hline \hline \multirow{2}{*}{$r$} & \multicolumn{3}{|c}{$P$} \\
\cline { 2 - 4 } & $(\mathrm{a})$ & $(\mathrm{b})$ & $(\mathrm{c})$ \\
\hline 2.831 & 0 & 0 & 0 \\
2.811 & 5.0 & 5.0 & 5 \\
2.794 & 10.0 & 10.0 & 10 \\
2.780 & 14.7 & 14.9 & 15 \\
2.764 & 19.7 & 19.8 & 20 \\
2.750 & 24.6 & 24.7 & 25 \\
2.738 & 29.4 & 29.8 & 30 \\
\hline percentage deviation & $2 \%$ & $0.67 \%$ & \\
at 30 kbar & & &
\end{tabular}


constant, electronic mass, and energy term values. Moreover, the exponential factors are different in different pair interactions, whereas in the Born-Mayer potential a common value of hardness parameters has been taken for the cation-anion, cation-cation, and anion-anion interactions. This is not justified for the reasons discussed by Shanker and Agrawal [11]. If one takes unequal values of hardness parameters for the crystal, then the number of parameters becomes too large to be determined from the input data for the lattice parameter and bulk modulus. Thus, the Harrison potential form is superior to the Born-Mayer exponential forms.

Finally, it should also be mentioned that the formulations proposed in the present study is of general nature and therefore its application may be extended to different classes of solids. The results of the present investigation also lead to the understanding of the equation of state based on the microscopic details of the theory of interionic potentials.

\section{Acknowledgments}

This work was supported by the Anhui Provincial Natural Science Foundation (No. 090416235 and No. 11040606 M07), the Talent Foundation of High Education of Anhui Province for Outstanding Youth (2011SQRL014), and the Youth Science Foundation of Anhui University (No. 2009QN024B).

\section{References}

[1] O.L. Anderson, Equations of State of Solids for Geophysics and Ceramic Science, Oxford University Press, New York 1995.

[2] L.L. Boyer, Phys. Rev. B 27, 1271 (1983).
[3] M. Kumar, Physica B 205, 175 (1995).

[4] R.K. Pandey, J. Phys. Chem. Solids 59, 1157 (1998).

[5] Q. He, Z.T. Yan, Phys. Status Solidi B 223, 767 (2001).

[6] Z.H. Fang, Phys. Status Solidi B 241, 2886 (2004).

[7] Manoj Kumar, M. Kumar, Physica B 403, 3672 (2008).

[8] W.A. Harrison, Phys. Rev. B 23, 5230 (1981).

[9] M. Born, K. Huang, Dynamical Theory of Crystal Lattices, Oxford University Press, Oxford 1954.

[10] S.K. Srivastava, S.K. Sharma, P. Sinha, J. Phys. Chem. Solids 70, 255 (2009).

[11] J. Shanker, G.G. Agrawal, Phys. Status Solidi B 123, 11 (1984).

[12] M.P. Tosi, in: Solid State Physics, Vol. 16, Eds. F. Seitz, D. Thurnbull, H. Ehrenreich, Academic Press, New York 1964, p. 1.

[13] O.L. Anderson, D.G. Isaak, in: Mineral Physics and Crystallography, A Handbook of Physical Constants, AGU Reference Shelf 2, Washington (DC) 1995, p. 64.

[14] R. Boehler, G.C. Kennedy, J. Phys. Chem. Solids 41, 517 (1980).

[15] T. Yagi, J. Phys. Chem. Solids 39, 563 (1978).

[16] S.N. Vaidya, G.C. Kennedy, J. Phys. Chem. Solids 32, 951 (1971).

[17] K.K. Srivastava, H.D. Merchant, J. Phys. Chem. Solids 34, 2069 (1973).

[18] J.L. Tallon, J. Phys. Chem. Solids 41, 837 (1980).

[19] X.Q. Deng, Z.T. Yan, High Temp. High Press. 34, 387 (2002).

[20] F.D. Stacey, Rep. Prog. Phys. 68, 341 (2005). 